\title{
Application of the Bayesian analysis to the Modified Rutherford Equation for NTM stabilisation
}

\author{
L. Urso ${ }^{1}$, R. Fischer ${ }^{1}$, A. Isayama ${ }^{2}$, ASDEX Upgrade Team ${ }^{1}$ and JT-60 \\ Team $^{2}$ \\ ${ }^{1}$ MPI für Plasmaphysik, Boltzmannstr. 2, D-85748 Garching, EURATOM-Association, \\ Germany \\ 2 Japan Atomic Energy Agency, Naka, Ibaraki 311-0193, Japan \\ E-mail: laura.urso@ipp.mpg.de
}

\begin{abstract}
The determination of the free parameters present in the modified Rutherford equation (MRE), which is routinely used for studying the physics of Neoclassical Tearing Mode (NTM) stabilisation, is addressed by making use of the Bayesian probability theory. The evaluation of the free coefficients is particularly sensitive to the assumptions used in the modelled equation, to the correlation of various physical parameters, and to the uncertainties of the experimental measurements. A probabilistic method was applied for the consistent evaluation of the coefficients and their uncertainties using a large database of discharges and by considering the correlations and the uncertainties of the multiple physical quantities present in the MRE. The estimated values and uncertainties of the coefficients are related to the precise determination of the minimum amount of ECCD power necessary to stabilise NTMs in ITER.
\end{abstract}

\section{Introduction}

Neoclassical Tearing Modes (NTMs) are a type of resistive instability which limit the performance of present tokamaks and are expected to be highly detrimental for the International Thermonuclear Experimental Reactor (ITER). They are present in the plasma in the form of magnetic islands whose saturation and decay are modelled by the Modified Rutherford Equation (MRE). The MRE is a differential equation which describes the behaviour of the magnetic island width $W$. By applying the MRE to present devices it is possible to extrapolate the NTM behaviour and stabilisation requirements to ITER. In fact, in ITER it is of major interest to predict the behaviour of NTMs and to quantify the amount of ECRH power in the form of current drive necessary to stabilise them.

The main drive which allows the island to grow is the missing bootstrap current $I_{\mathrm{BS}}$ inside the island [1]. The bootstrap current is present in a toroidal plasma and is due to the combined effects of trapped particles and pressure gradient [2]. Stabilisation of NTMs is achieved by means of Electron Cyclotron Current Drive (ECCD) which counteracts the loss of $I_{\mathrm{BS}}$ by injecting inside the island ECCD current $I_{\mathrm{ECCD}}$ [3]. Other stabilising effects are given by the influence of the island on the equilibrium current profile and the favourable tokamak curvature [4]. Free coefficients in the MRE account for uncertainties and approximations used to derive 
the analytical model (e.g. simplifications in the geometry and simplified physics). For the MRE used in this work two coefficients are introduced, namely $c_{\text {sat }}$ and $c_{\text {stab }}$ (equation [1]). These fitting coefficients are on the order of unity. It is assumed that they have the same meaning for all devices and for all types of NTMs (e.g. for every helicity $(m, n)$ where $m$ and $n$ are the poloidal and toroidal mode numbers, respectively). They have been calculated for a set of NTM stabilisation discharges from the ASDEX Upgrade [5] and JT-60U devices [6]. The estimation of their values depend on many plasma parameters which are correlated among themselves and are deteriorated with measurement uncertainties.

Considering the multiple correlations and uncertainties the question arises how consistently one can estimate the uncertainties on $c_{\text {sat }}$ and $c_{\text {stab }}$. Often the discussion on the error bars of these coefficients is neglected due to its complicated nature. On the other hand, the evaluation of the error bars on $c_{\text {sat }}$ and $c_{\text {stab }}$ is directly related to the precise determination of the minimum amount of ECCD power necessary to stabilise NTMs in ITER.

In order to approach this task, an error analysis using a probabilistic method was developed. The probabilistic approach based on Monte-Carlo sampling of physical quantities from correlated probability distributions generalises the frequently used Gaussian error propagation technique. Applying Gaussian error propagation to the MRE is actually not feasible if all dependencies and uncertainties have to be considered. The proposed method is based on Bayesian Probability Theory (BPT) [7] which provides a framework for scientific reasoning from uncertain data. BPT allows one to combine any sort of data or information [8], [9]. Uncertainty or lack of knowledge as well as correlated information is quantified with probability distribution functions (PDF). Non-Gaussian error propagation of multiple uncertainties is inherent to BPT and can be easily performed with Monte-Carlo sampling from PDFs.

The main focus is to investigate the sensitivity of the evaluation of $c_{\text {sat }}$ and $c_{\text {stab }}$ on the measurements of the physical quantities. A thorough determination of all relevant measurement uncertainties and of the correlation of the plasma quantities is provided. The result of the probabilistic method is a two-dimensional PDF for the two coefficients of the MRE which allows one to estimate the most probable values, the individual uncertainties and the correlation between the coefficients. A sensitivity study shows which physical parameters are most crucial for a reliable estimation of the coefficients and for extrapolation to ITER.

In section 2 the MRE is outlined. The NTM stabilisation experiments are described in section 3. In section 4 the measured and correlated (dependent) quantities are specified together with their respective uncertainties. In section 5 the standard least-squares minimisation technique and the new probabilistic method are depicted. The results for $c_{\text {sat }}$ and $c_{\text {stab }}$ and a sensitivity study are shown in section 6 . Finally, section 7 summarises and concludes. 


\section{The Modified Rutherford Equation}

A complete form of the MRE is given by [10]

$$
\begin{aligned}
& \frac{\tau_{\mathrm{s}}}{r_{\text {res }}} \frac{d W}{d t}=-r_{\text {res }} \Delta^{\prime}+c_{s a t}\left[r_{\text {res }} 3.17 \mu_{0} L_{\mathrm{q}} \frac{j_{\mathrm{BS}}}{B_{\mathrm{pol}}}\left(\frac{W}{W^{2}+W_{\mathrm{d}}^{2}}+\frac{W}{W^{2}+28 W_{\mathrm{b}}^{2}}\right)\right. \\
& \left.-\frac{r_{\text {res }} 6.35 \mu_{0} D_{R}}{\sqrt{W^{2}+0.65 W_{d}^{2}}}\right]-c_{\text {stab }} 16 \sqrt{\pi} \mu_{0} r_{\mathrm{res}} L_{\mathrm{q}} \frac{j_{\mathrm{ECCD}}}{B_{\mathrm{pol}}} d \frac{\eta_{\mathrm{ECCD}}}{W^{2}}
\end{aligned}
$$

where $W$ is the full magnetic island width, $r_{\text {res }}$ is the radius at the rational surface, $\tau_{\mathrm{s}}=$ $1.22 r_{\text {res }}^{2} / \eta_{N C}$ is the resistive time and $\eta_{N C}$ is the neoclassical resistivity. The different terms describe the stabilising ( - sign) and destabilising ( + sign) contributions of the physical mechanisms. The first term on the right-hand side is the so-called $\Delta^{\prime}$-term which is modelled for a cylinder as $-r_{\text {res }} \Delta^{\prime}=-m$ [12] in case of a $(2,1)$ NTM and $-r_{\text {res }} \Delta^{\prime}(W)=-1.97 \frac{r_{\text {res }}}{a}(1+$ $23.1 \frac{W}{a}$ for a $(3,2)$ NTM. In the latter case, in fact, a fit including the island width dependence has been available [11]. The cylindrical assumption is frequently used although it does not take into account important effects such as the toroidal coupling between different harmonics. Although the $\Delta^{\prime}$-term affects systematically the estimates of $c_{\text {sat }}$ and $c_{\text {stab }}$, a thorough treatment of the $\Delta^{\prime}$-term variants is beyond the scope of this work. For the studied NTMs, in fact, the $\Delta^{\prime}$-term is a negative quantity which has therefore a stabilising effect on the evolution of the magnetic island.

The second term on the right-hand side is the bootstrap term where $L_{\mathrm{q}}=\frac{q}{q^{\prime}}$ is the gradient scale length of the safety factor profile $\mathrm{q}, j_{\mathrm{BS}}$ is the local value of the bootstrap current density, and $B_{\text {pol }}$ is the mean poloidal field at the resonant surface. The bootstrap term has a destabilising effect on the evolution of the magnetic island. The bootstrap current density is proportional to $j_{\mathrm{BS}} \propto p / L_{\mathrm{p}}$, where $p=2 n_{\mathrm{e}}\left(T_{\mathrm{e}}+T_{\mathrm{i}}\right)$ is the plasma pressure and $n_{\mathrm{e}}$ is the electron density for which it is assumed that the ion density $n_{\mathrm{i}}=n_{\mathrm{e}} . T_{\mathrm{e}}$ and $T_{\mathrm{i}}$ are the electron and ion temperatures, respectively. $L_{\mathrm{p}}=p / p^{\prime}$ is the pressure gradient scale length. The bootstrap term also includes the effect of incomplete flattening of the temperature profile modelled with the threshold island width $W_{\mathrm{d}}=5.1 \sqrt{\frac{R_{\mathrm{maj}} L_{\mathrm{q}} q}{m}}\left(\frac{\chi_{\perp}}{\chi_{\|}}\right)^{1 / 4}$ where the perpendicular thermal conductivity is expressed as $\chi_{\perp}=\frac{3.25 \sqrt{T_{\mathrm{i}}}}{B_{\mathrm{T}}^{2}} \frac{\sqrt{A}}{Z^{2}}$ with $B_{\mathrm{T}}$ being the toroidal magnetic field, $a$ being the minor radius, $A$ the atomic number and $Z$ the charge. The parallel thermal conductivity is given by $\chi_{\|}=\frac{\chi_{\mathrm{Sp}}}{\sqrt{1+\left(3.16 \cdot 380 / L_{\|}\right)}}$with $\chi_{\mathrm{Sp}}=\frac{26.4 \sqrt{\pi} T_{\mathrm{e}}^{5 / 2} \varepsilon_{0}^{2}}{2 m_{\mathrm{e}} \varepsilon^{3 / 2} \ln \Lambda n_{\mathrm{e}}}$ being the Spitzer conductivity from the classical transport theory [12]. $L_{\|}=\frac{R_{\mathrm{maj}} L_{\mathrm{q}}}{4 n W}$ is the connection length, $R_{\text {maj }}$ is the major radius and $\Lambda$ is the Coulomb logarithm. The bootstrap term also includes the beneficial effect of trapped particles sustaining the bootstrap current inside the island which is modelled by $W_{\mathrm{b}}=\varepsilon^{1 / 2} \rho_{\mathrm{pi}}$ with $\rho_{\mathrm{pi}}=\frac{\sqrt{2 m_{\mathrm{i}} k_{\mathrm{B}} T_{\mathrm{i}}\left(r_{\text {res }}\right)}}{e B_{\mathrm{pol}}\left(r_{\mathrm{res}}\right)}$ being the ion poloidal gyro-radius, $m_{\mathrm{i}}$ the ion mass, $e$ the electron charge and $k_{\mathrm{B}}$ being the Boltzmann constant. The curvature term is written in terms of $D_{\mathrm{R}}=-\frac{2 p^{\prime}\left(1-q^{2}\right) q^{2}}{r_{\mathrm{res}} B_{\mathrm{T}}^{2} q^{\prime 2}}$ which is the so-called resistive interchange parameter.

The third term models the external injection of ECCD current $j_{\mathrm{ECCD}}$ which is given by the peak value $j_{\mathrm{ECCD}, 0}$ of the ECCD Gaussian current density profile $j_{\mathrm{ECCD}}=$ 
$j_{\mathrm{ECCD}, 0} e^{-C\left(\frac{r-r_{\text {res }}}{d}\right)^{2}}$ where $d$ is the full deposition width at half maximum, $C=4 \ln 2$ and $j_{\mathrm{ECCD}, 0}$ is calculated from the total $I_{\mathrm{ECCD}}$ current. $\eta_{\mathrm{ECCD}}$ describes the efficiency of ECCD considering the relative alignment of the island width and the ECCD beam and the relative width of the island size $W$ to the deposition width $d$.

The above formulas show that each term contributing to the MRE includes dependencies among the various physical quantities and many of the dependencies are non-linear in nature. The form of the MRE used in this work does not take into account the polarisation current effect, or other possible terms describing viscosity, flow shear, or coupling between surfaces and the wall. In fact, at the present time a single complete form of the MRE does not exist. However, the main attempt of this work is to study the uncertainty of the fitting parameters due to the quantities included in the present MRE. The depicted probabilistic method is not restricted to the present MRE but can be easily applied to any sophistication of the MRE.

The evolution of the magnetic island can be analysed by considering the stationary solutions $(d W / d t=0)$ of equation (1) and by using its phase diagram $(W, d W / d t)$. The experimental database allows us to determine all physical parameters present in equation (1) as well as to observe directly the island width evolution from the magnetic perturbation signal $(W \sim \sqrt{\delta B})$.

\section{NTM stabilisation experiments and database}

For both devices, ASDEX Upgrade and JT-60U, a typical NTM stabilisation experiment consists of three phases: A first phase, in which an NTM is destabilised by increasing the pressure drive $(\beta)$. A second phase, in which a saturated mode (of width $W_{\text {sat }}$ and $d W / d t=0$ ) is allowed to form and a third phase, in which the ECCD power $P_{\mathrm{ECRH}}$ is applied to generate a highly localised current $I_{\mathrm{ECCD}}$ in the region where the resonant surface $r_{\text {res }}$ is located. The discharges used in this work consist of 5 different sets of $(3,2)$ and $(2,1)$ NTMs successfully stabilised with ECCD. They are carried out in different experimental campaigns both at ASDEX Upgrade and JT-60U. Two examples for NTM stabilisation discharges are shown in figure 1 for ASDEX Upgrade (left panel) and JT-60U (right panel), respectively. The measured island width $W$, derived from the Mirnov coil diagnostic, will be compared to the simulation from the MRE as shown in figure 9. Table 1 summarises the global plasma parameters together with the geometrical factor $q_{95}=s \frac{a^{2} B_{T}}{R_{\text {maj }} I_{p}}$ and the shaping factor $s$ for the five different sets of NTM stabilisation discharges at ASDEX Upgrade and JT-60U. The difference in the mean values for $s$ between the two machines is about $30 \%$ whereas within the ASDEX Upgrade and JT-60U databases the differences in the geometrical set-ups between $(3,2)$ NTM and $(2,1)$ NTM discharges are smaller. The saturated island widths are determined from the contour plots of the electron temperature measurements $T_{\mathrm{e}}(R)$ from the ECE diagnostics. Despite the good quality of the ECE data a width uncertainty of $1-2 \mathrm{~cm}$ is still present as shown in figure 2. The uncertainty depends on the limited spatial resolution of the ECE channels as well as on the temporal resolution of the data acquisition system, which is sufficient to resolve the frequency of the mode. An example of the kinetic profiles $T_{\mathrm{e}}, T_{\mathrm{i}}$, and $n_{\mathrm{e}}$, is given in the figures 3 and 4 for ASDEX Upgrade and JT-60U, respectively. 

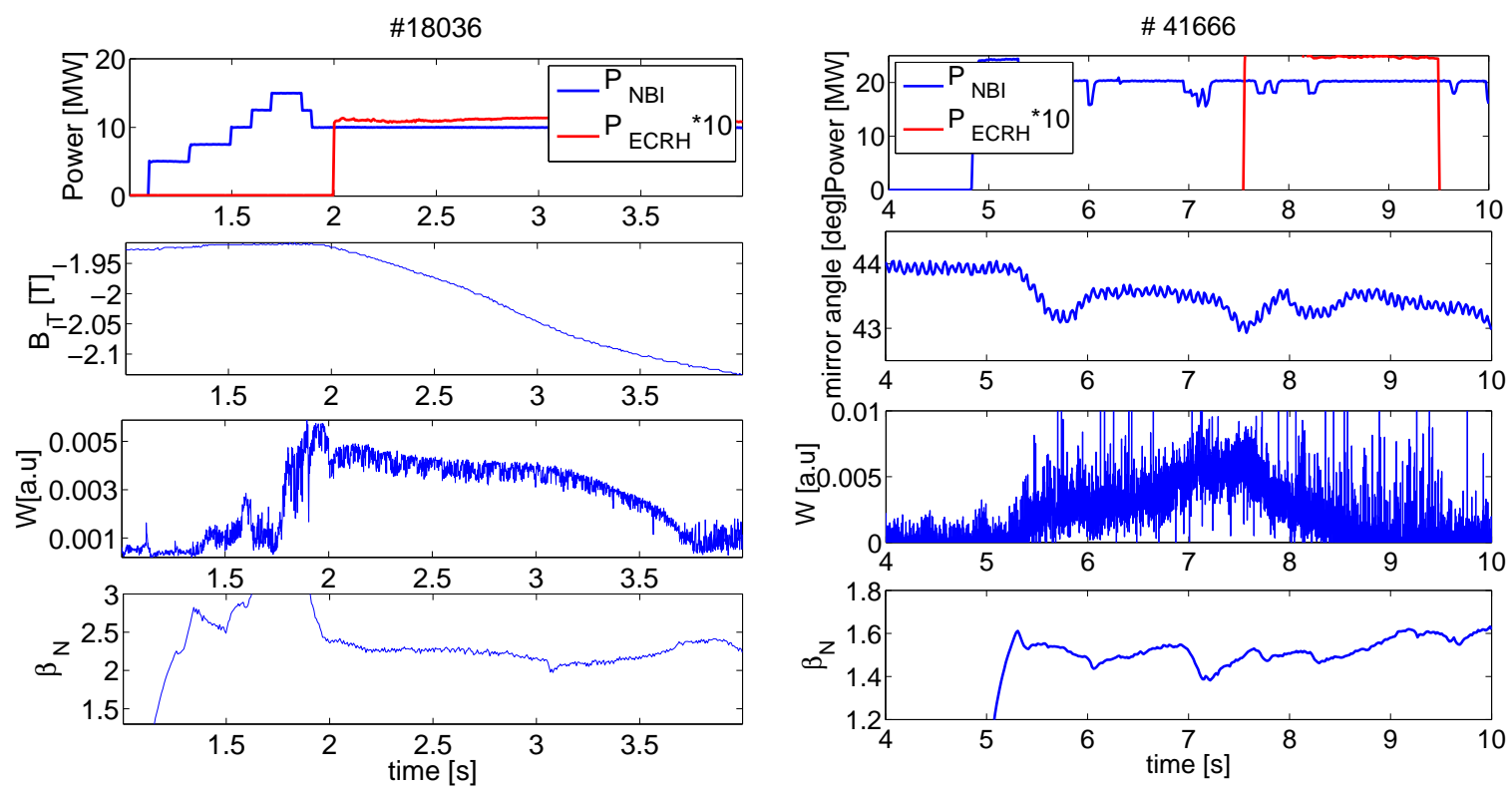

Figure 1. Typical time slices for NTM stabilisation experiments for \#18036 with $\beta_{\mathrm{N}} \sim 2.2$ (ASDEX Upgrade) and for $\# 41666$ with $\beta_{\mathrm{N}} \sim 1.5$ (JT-60U). The NBI power $P_{\mathrm{NBI}}$ is increased until the NTM is destabilised. Once the mode is triggered, $P_{\mathrm{NBI}}$ is decreased to a level for which the available $P_{\mathrm{ECRH}}$ power is sufficient to effectively suppress the mode. As a consequence $\beta_{\mathrm{N}}$ increases.

\begin{tabular}{c|cccc|c}
\hline NTM & $I_{\mathrm{p}}[\mathrm{MA}]$ & $B_{\mathrm{T}}[\mathrm{T}]$ & $\frac{a^{2}}{R_{\mathrm{maj}}}$ & $q_{95}$ & $s$ \\
\hline (2,1) NTM ASDEX Upgrade & 0.8 & 1.85 & 0.14 & 4.4 & 14.2 \\
(2,1) NTM - a JT-60U & 1.5 & 3.7 & 0.19 & 3.9 & 8.3 \\
(2,1) NTM - b JT-60U & 0.85 & 1.7 & 0.19 & 3.5 & 10.0 \\
(3,2) NTM ASDEX Upgrade & 0.8 & 2.1 & 0.14 & 4.8 & 12.5 \\
(3,2) NTM JT-60U & 1.5 & 3.7 & 0.19 & 3.75 & 8.0 \\
\hline
\end{tabular}

Table 1. Set of global parameters for JT-60U and ASDEX Upgrade for $(2,1)$ and $(3,2)$ NTMs and resulting shaping factor $s$

The evaluation of quantities like the bootstrap current $j_{\mathrm{BS}}$ is strongly affected by the gradient length scales of the profiles, which are shown for both devices in figure 5 . The $T_{\mathrm{e}}$ and $T_{\mathrm{i}}$ profiles are typically stiff for H-mode discharges. In contrast, the shape of the density profile strongly depends on the collisionality. For different collisionalities, the gradient length can change up to a factor of 2-3. Therefore, the profile of the density gradient length $L_{\mathrm{n}}$ is most uncertain. The absolute value of $L_{\mathrm{n}}$ interpolated at the resonant surface of the mode (dotted lines) is larger at ASDEX Upgrade than at JT-60U by almost a factor of 2. This is in agreement with the expectation that for lower collisionality (JT-60U case) the density profile is more peaked.

Figure 6 shows the safety factor profiles which are determined from equilibrium calculations. In the core of the plasma, MHD activity can help to constraint the q-profile. 


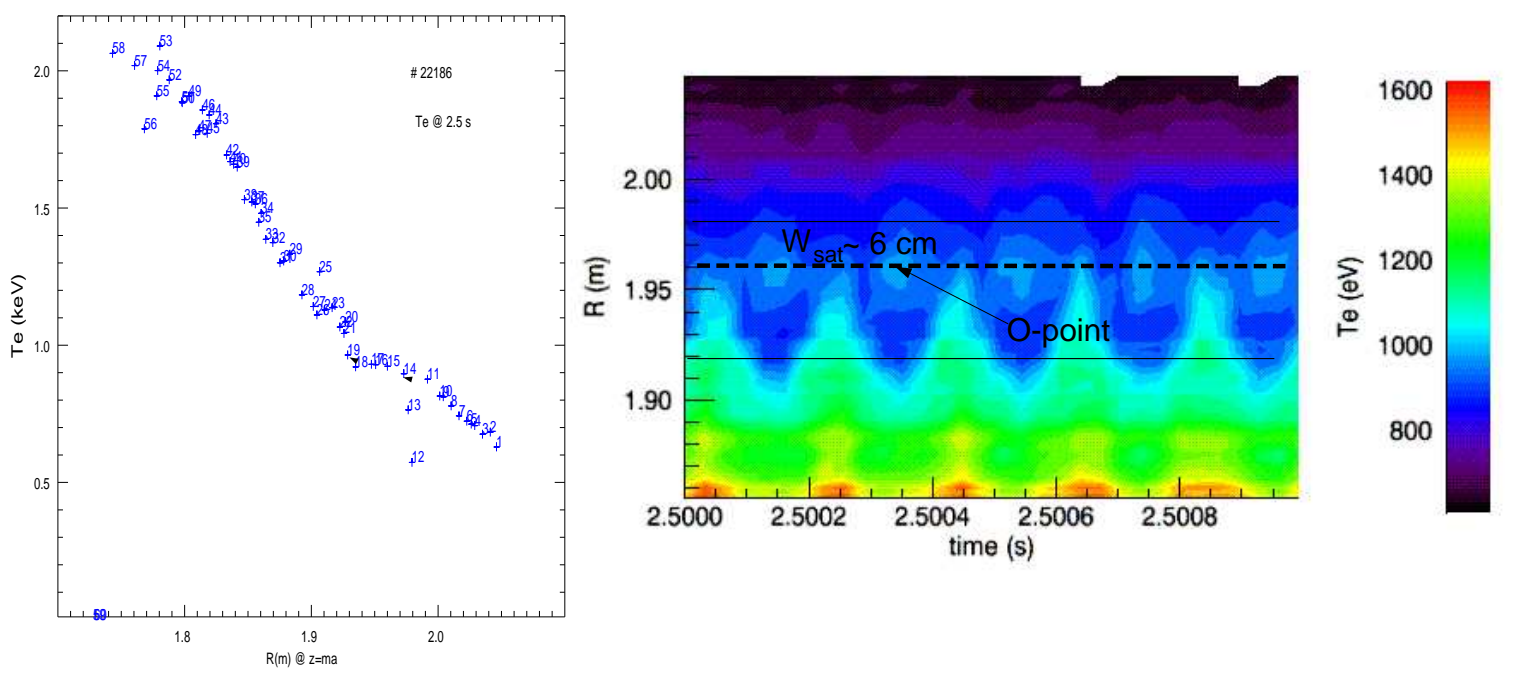

Figure 2. Electron temperature profile for \#22186 together with the contour plot which shows the $(2,1)$ magnetic island chain and the asymmetric shape of the magnetic island. The O-point of the island is at $R=1.96 \mathrm{~m}$ which corresponds to $r_{\text {res }}=0.25 \mathrm{~m}$
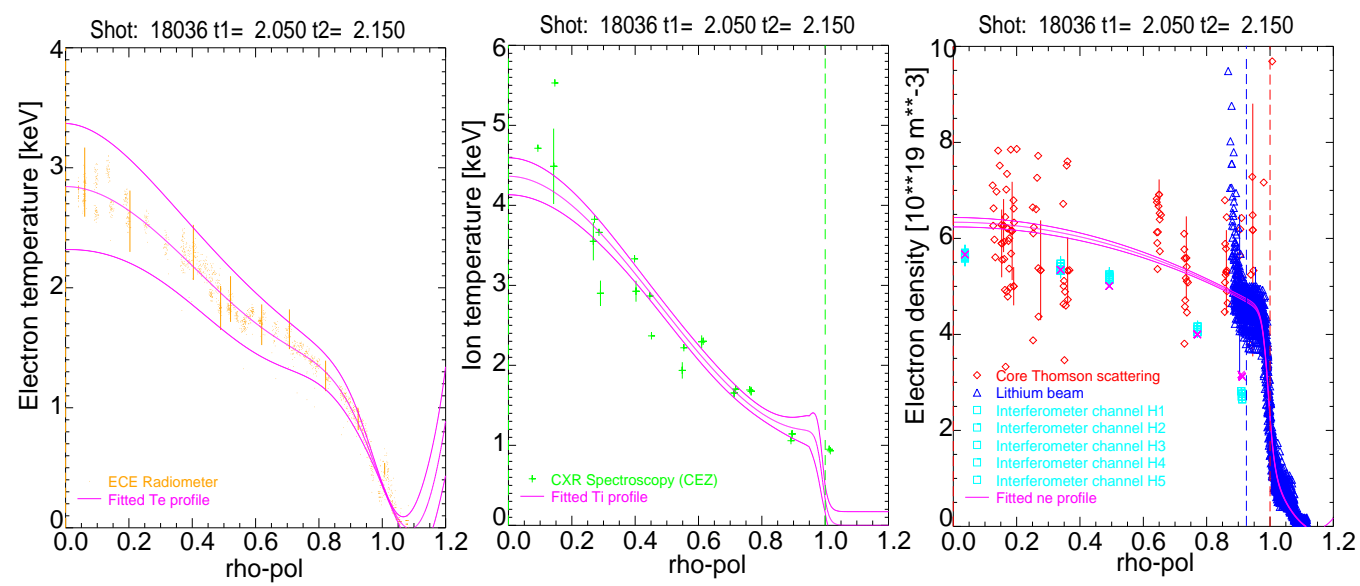

Figure 3. Kinetic profiles for ASDEX Upgrade: The electron temperature profile is fitted using ECE data (left), the ion temperature profile is fitted using CXRS data (centre), and the density profile (right) is fitted by combining Thomson scattering data (up to $\rho_{\text {pol }}=1.0$ ), Lithium beam data (starting from $\rho_{\text {pol }}=0.93$ ), and by matching the integrated values of the data from the 5 interferometry channels.

However, the determination of the safety factor profile is relying on the Motional stark effect diagnostic. The estimate of the ECCD current density and the deposition profile depends not only on the kinetic calculations but also on the experimental set-up which determines the width of the deposited profile. An example is shown in figure 7: $j_{\mathrm{BS}}$ is steeper at the edge of ASDEX Upgrade because of the steeper pressure gradient. Globally, $j_{\mathrm{BS}}$ is larger for ASDEX 

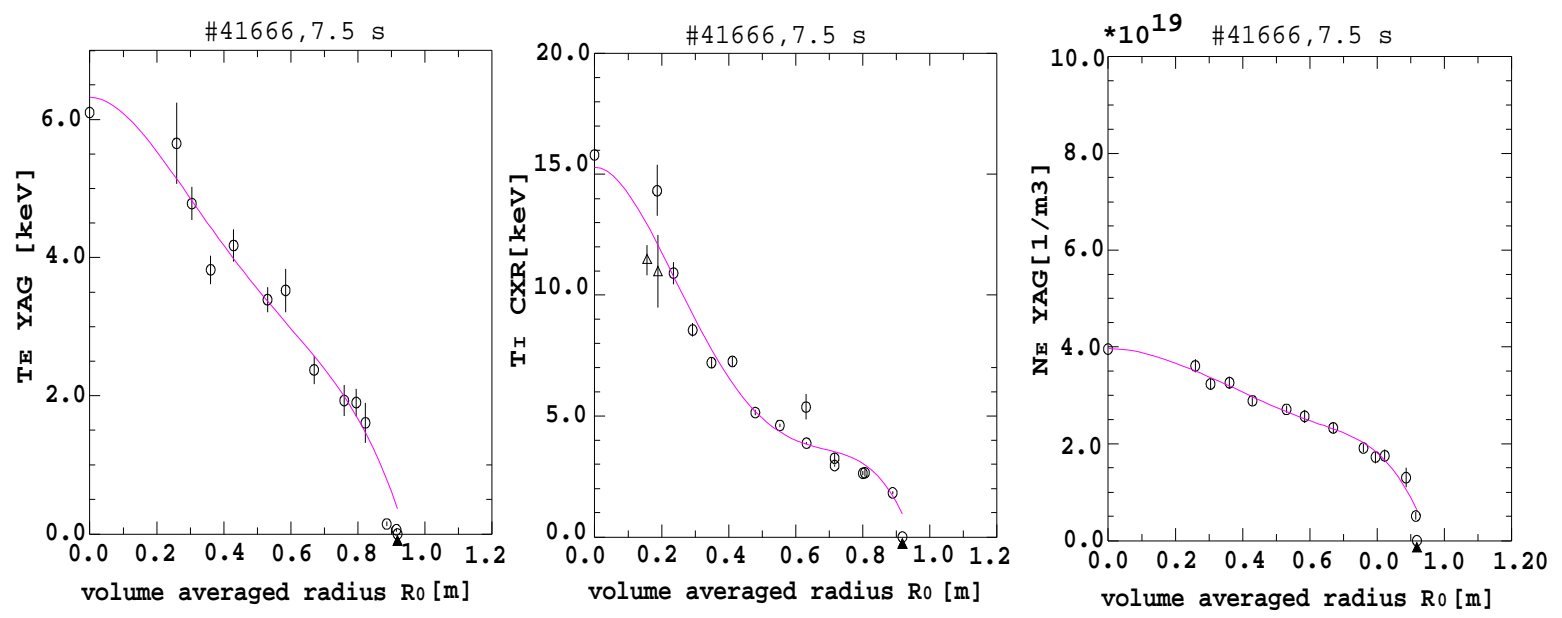

Figure 4. Kinetic profiles for JT-60U plotted versus the volume averaged minor radius $R_{0}$. The electron temperature (left) and density profiles (right) are fitted to Thomson scattering data whereas the ion temperature profile is fitted using CXRS data (centre).
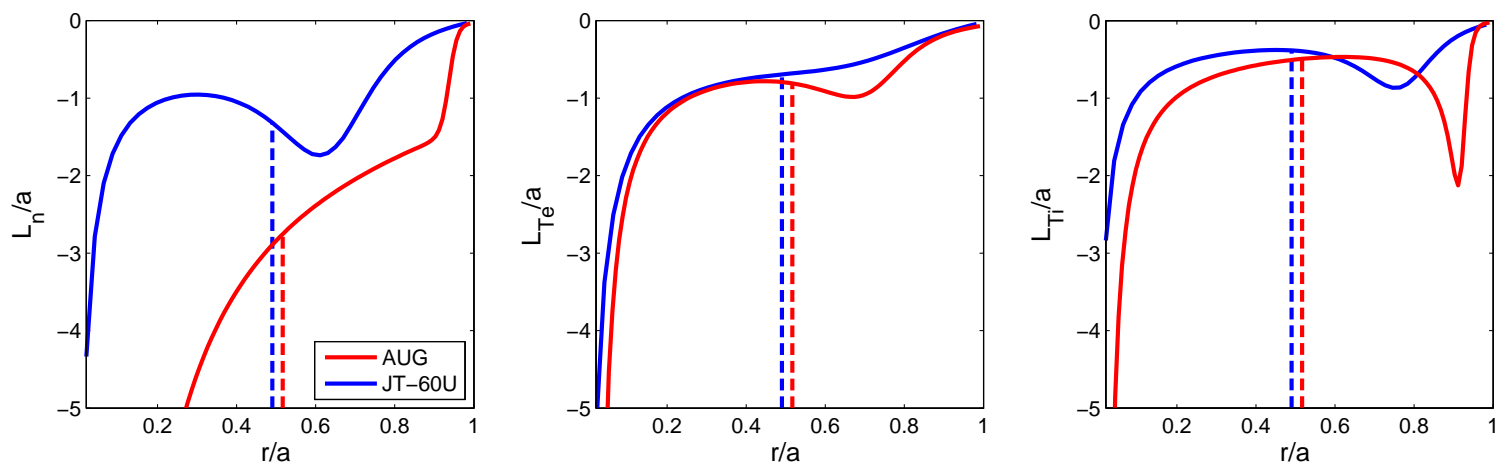

Figure 5. Gradient lengths of the kinetic profiles for JT-60U and ASDEX Upgrade. The dotted lines depict the location of the resonant surface of the mode

Upgrade than for JT-60U as the bootstrap current density mainly depends on the value of $\beta$, which is larger for the ASDEX Upgrade discharge. The $j_{\mathrm{ECCD}}$ current is characterised by a Gaussian profile and the mismatch between the mode location (blue dotted line) and the injection location (red dotted line) indicates the presence of a possible misalignment between the centre of the island and the ECCD beam.

\section{Values, multiple uncertainties, boundaries and correlations in physical quantities}

In general, the two fitting coefficients depend on the plasma quantities mentioned in the previous section in a non-linear way:

$$
\begin{aligned}
& c_{\mathrm{sat}}=c_{\mathrm{sat}}\left(W_{\mathrm{sat}}, L_{\mathrm{q}}, j_{\mathrm{BS}}, \ldots . .\right) \\
& c_{\mathrm{stab}}=c_{\mathrm{stab}}\left(W_{\mathrm{sat}}, L_{\mathrm{q}}, j_{\mathrm{BS}}, j_{\mathrm{ECCD}}, d, \ldots . .\right)
\end{aligned}
$$



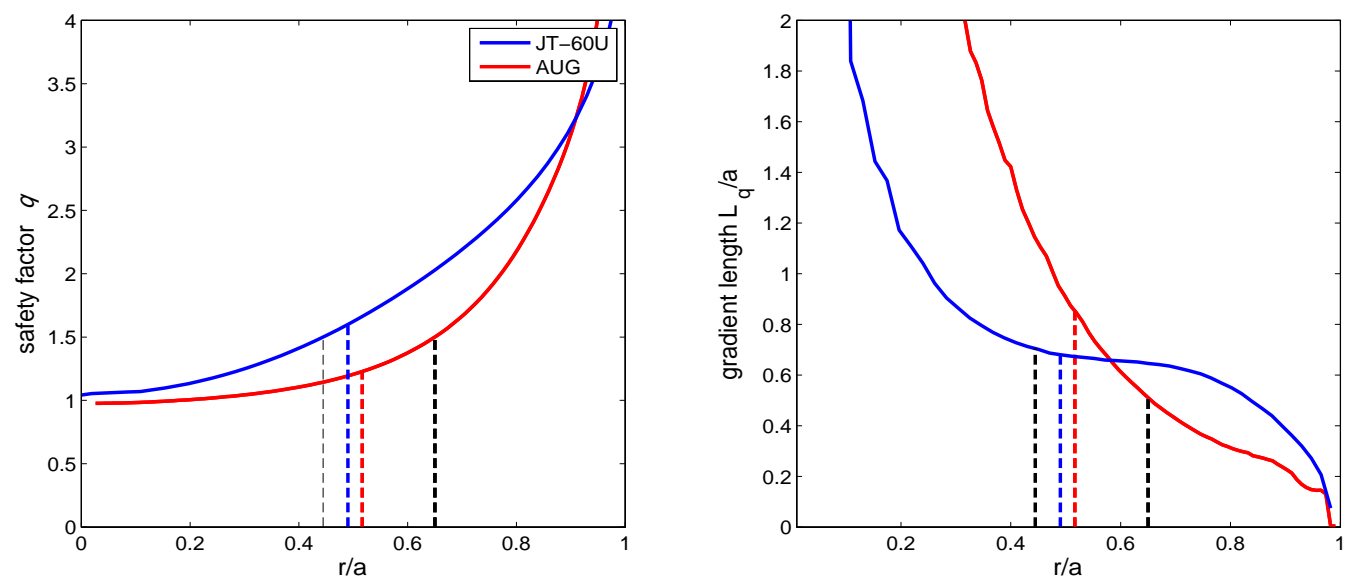

Figure 6. Safety factor profile and gradient length $L_{\mathrm{q}}$ for JT-60U and ASDEX Upgrade discharges. The local values of the mode position (from the ECE measurement) and of the $\mathrm{q}=1.5$ surface (from the equilibrium) are indicated with dotted lines.
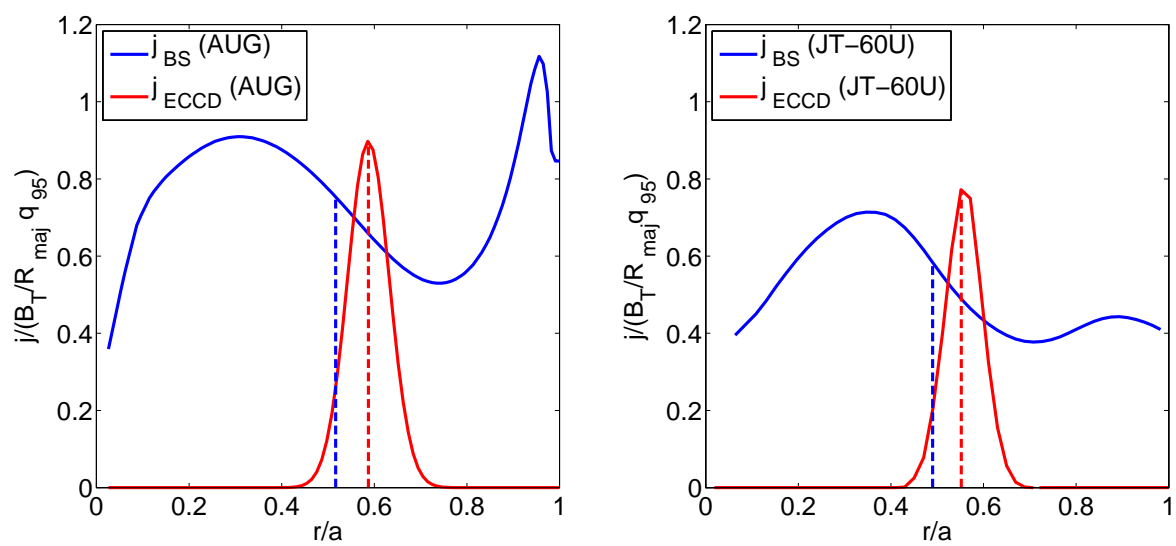

Figure 7. Bootstrap current density profile $j_{\mathrm{BS}}$ for ASDEX Upgrade and JT-60U discharges together with the Gaussian profile of the injected $j_{\text {ECCD }}$ current density of width $d$ for discharge \#18036 (left) with $\beta_{\mathrm{N}} \sim 2.2$ and discharge \#41666 (right) with $\beta_{\mathrm{N}} \sim 1.5$ both normalised to $\frac{B_{\mathrm{T}}}{R_{\text {maj }} q_{95}}$.

\subsection{Values}

In order to determine $c_{\text {sat }}$ and $c_{\text {stab }}$ with their error bars, the plasma parameters which need to be considered at saturation time $t_{\mathrm{sat}}$ and at stabilisation time $t_{\mathrm{ECCD}}$ are the following:

(i) the radial location of the mode $r_{\text {res }}$ and the saturated island width $W_{\text {sat }}$ are measured from the flattening of the electron temperature profile

(ii) the electron density $n_{\mathrm{e}}$, electron temperature $T_{\mathrm{e}}$ and ion temperature $T_{\mathrm{i}}$ profiles are obtained from the combination of different diagnostics

(iii) the safety factor profile $q$ and the gradient scale length $L_{\mathrm{q}}=q / q^{\prime}$ are calculated using the equilibrium current profile 
(iv) the bootstrap current density profile $j_{\mathrm{BS}}$ is calculated with a transport code using the kinetic profiles $\left(n_{\mathrm{e}}, T_{\mathrm{e}}, T_{\mathrm{i}}\right)$

(v) the ECCD current density profile $j_{\mathrm{ECCD}}$ and the ECCD deposition width $d$ are calculated with a ray-tracing code using the kinetic profiles

(vi) the neoclassical resistivity $\eta_{\mathrm{NC}}$ is calculated with a transport code using the kinetic profiles

(vii) the gradient scale lengths of the kinetic profiles $L_{\mathrm{Te}}, L_{\mathrm{Ti}}, L_{\mathrm{ne}}$ are obtained using the derivative over the minor radius of the fitted kinetic profiles

(viii) the poloidal magnetic field $B_{\mathrm{pol}}$ is calculated from the equilibrium reconstruction and the toroidal magnetic field $B_{\mathrm{T}}$ is obtained from the magnetic coils' measurements

(ix) the efficiency function $\eta_{\text {ECCD }}$ of the ECCD injection is calculated analytically [15].

(x) the growth rate $\left.\frac{d W}{d t}\right|_{t_{\mathrm{ECCD}}}$ and the decay of the island width $W_{\mathrm{ECCD}}$ are obtained from an integration of the MRE.

The dependent parameters $p, L_{\mathrm{p}}, \chi_{\perp}, \chi_{\|}, \rho_{\mathrm{pi}}, L_{\|}, D_{\mathrm{R}}, W_{\mathrm{b}}, W_{\mathrm{d}}, \tau_{\mathrm{s}}$ are calculated from the measured quantities $r_{\text {res }}, L_{\mathrm{q}}, W_{\mathrm{sat}}, n_{\mathrm{e}}, T_{\mathrm{e}}, T_{\mathrm{i}}, L_{\mathrm{ne}}, L_{\mathrm{Te}}, L_{\mathrm{Ti}}, B_{\mathrm{pol}}, B_{\mathrm{T}} \cdot j_{\mathrm{ECCD}}$ and $d$ are obtained from beam tracing calculations and $j_{\mathrm{BS}}$ and $\eta_{\mathrm{NC}}$ from transport modelling.

\subsection{Multiple uncertainties}

The quantities shown in the previous section can be split into two groups where one group contains quantities with significant uncertainties and the second group quantities where the uncertainties are negligible. The first group with values with significant uncertainties comprises $W_{\mathrm{sat}}, W_{\mathrm{ECCD}}, d W / d t_{t_{\mathrm{ECCD}}}, \eta_{\mathrm{ECCD}}, n_{\mathrm{e}}, T_{\mathrm{e}}, T_{\mathrm{i}}, j_{\mathrm{ECCD}}, j_{\mathrm{BS}}, d, L_{\mathrm{q}}, L_{\mathrm{ne}}, L_{\mathrm{Te}}, L_{\mathrm{Ti}}$. In the second group are $q, r_{\mathrm{res}}, \eta_{\mathrm{NC}}, B_{\mathrm{pol}}, B_{\mathrm{T}}$.

For a comprehensive error analysis proper estimates for the uncertainties of the various physical quantities are essential. In the Bayesian framework uncertainties are quantified with probability distribution functions. Although the Bayesian method can process any type of PDF only Gaussian distributions are used in this work. Gaussian distributions for the error statistic are the most uninformative distributions if only the mean (here $\langle\varepsilon\rangle=0$ ) and variance $\left(\left\langle\varepsilon^{2}\right\rangle=\sigma^{2}\right)$ are known. For the given physical quantities standard deviations for the error statistics could be estimated whereas higher moments of the error statistics are not known. If higher moments of the error statistics are relevant for the most sensitive physical quantities additional measurements are necessary to recover the precise statistics.

In general, for the quantities measured directly from a diagnostic the value given from the diagnostician is considered as the standard deviation, as for example in the case of the kinetic profiles (for which $\sigma \leq 7 \%$ for $T_{\mathrm{i}}$ and $T_{\mathrm{e}}$ and $\sigma \sim 10 \%$ for $n_{\mathrm{e}}$ both at ASDEX Upgrade and JT-60U have been assumed). For quantities which result from modelling codes (such as $j_{\mathrm{BS}}$ or $j_{\mathrm{ECCD}}$ ) or are calculated from derivatives (as the gradients $L_{\mathrm{q}}, L_{\mathrm{ne}}$ or $L_{\mathrm{Te}}$ ) estimating the proper uncertainty is not trivial since one should take into account the approximations in the relevant model, the numerical precision of the calculation, and the correlation in profiles. The uncertainties on the quantities $W_{\mathrm{ECCD}}, d W /\left.d t\right|_{t_{\mathrm{ECCD}}}$ and $\eta_{\mathrm{ECCD}}$ are small compared to, e.g., 
$W_{\text {sat }}$ and $L_{\mathrm{q}}$ because they do not vary much once the temporal evolution of the island width simulated from the MRE is determined from the experimental data. The standard deviations are estimated as a compromise between being too optimistic $(\sigma>5-10 \%)$ and being too pessimistic $(\sigma<50-60 \%)$ in the experimental data and modelling codes. Discharge specific uncertainties have not been considered as they have all been treated in the same way, with the same measurements conditions. The chosen values for the standard deviations are listed in table 2 .

Table 2. Standard deviations $\sigma$ given in percentage chosen for the statistical analysis

\begin{tabular}{c|c|c|c|c}
$L_{\mathrm{ne}}$ & $W_{\mathrm{sat}}, L_{\mathrm{q}}, j_{\mathrm{BS}}$ & $j_{\mathrm{ECCD}}, d, n_{\mathrm{e}}, L_{\mathrm{T}_{\mathrm{e}}}, L_{\mathrm{T}_{\mathrm{i}}}, \eta_{\mathrm{ECCD}},\left.\frac{d W}{d t}\right|_{\mathrm{ECCD}}$ & $T_{\mathrm{e}}, T_{\mathrm{i}}$ & $W_{\mathrm{ECCD}}$ \\
\hline $20 \%$ & $15 \%$ & $10 \%$ & $7 \%$ & $5 \%$
\end{tabular}

\subsection{Boundaries and correlations}

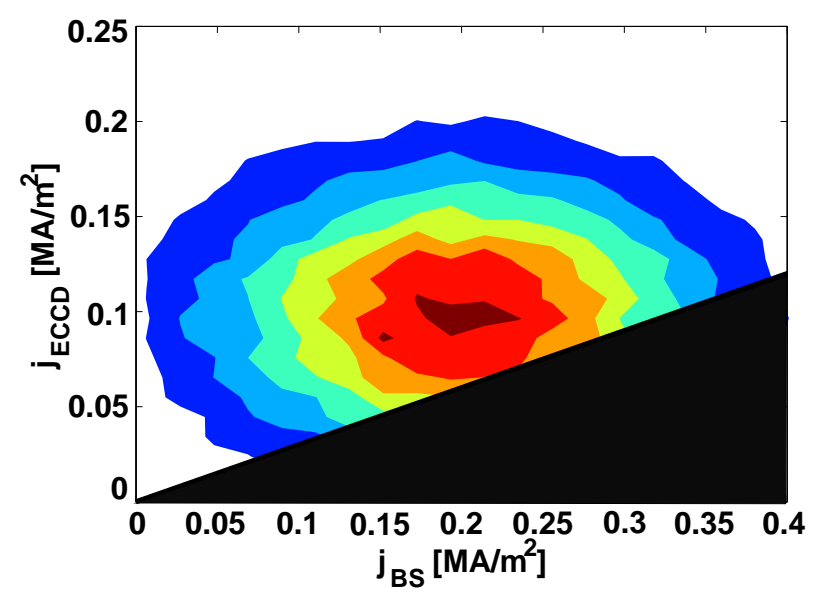

Figure 8. Probability distribution function for $j_{\mathrm{ECCD}}$ and $j_{\mathrm{BS}}$ considering the physics' boundary imposed. The randomisation of $j_{\mathrm{ECCD}}$ and $j_{\mathrm{BS}}$ satisfies the condition $\frac{j_{\mathrm{ECCD}}}{j_{\mathrm{BS}}}>0.3$ which, in the present database, is the marginal value which still allows for stabilisation.

The estimation of the MRE coefficients and their uncertainties is based on a Monte-Carlo method which randomises the physical quantities according to their uncertainty probability distributions. Physical boundaries on as well as correlations between the physical quantities can easily be incorporated into the PDFs. The physics boundaries are given by positivity or negativity $\left(L_{\mathrm{Te}}, L_{\mathrm{ne}}, d W /\left.d t\right|_{\mathrm{ECCD}}\right)$ constraints, a minimum value of $2 \mathrm{~cm}$ for the deposition width $d$, a minimum ratio $j_{\mathrm{ECCD}} / j_{\mathrm{BS}}$ for stabilisation of $\sim 0.3$ (as shown in figure 8 ), and a lower boundary for $W_{\text {sat }}$ of $W_{\mathrm{d}}$ or $W_{\mathrm{b}}$. Therefore, the PDFs of the physical quantities are normally distributed with cut-offs due to their constraints. 


\section{Determination of $c_{\mathrm{sat}}$ and $c_{\mathrm{stab}}$}

\section{1. $\chi^{2}$ approach to the Modified Rutherford equation}

The standard technique to evaluate $c_{\text {sat }}$ and $c_{\text {stab }}$ has been to measure the necessary physical quantities $\bar{B}=\left(W_{\text {sat }}, L_{\mathrm{q}}, \ldots\right)$ present in equation (1) at two specific time points $t_{1}=t_{\text {sat }}$ and $t_{2}=t_{\mathrm{ECCD}}$. For a statistical analysis the following quantities are defined:

$$
\begin{aligned}
& \xi_{0 i}=-r_{\text {res }} \Delta^{\prime}-\left.\frac{\tau_{\mathrm{s}}}{r_{\text {res }}}\left(\frac{d W}{d t}\right)\right|_{t_{\mathrm{i}}} \\
& \xi_{1 i}=r_{\text {res }}\left(\Delta_{\mathrm{BS}}^{\prime}+\Delta_{\mathrm{GGJ}}^{\prime}\right) \\
& \xi_{2 i}=r_{\text {res }} \Delta_{\mathrm{ECCD}}^{\prime}
\end{aligned}
$$

where $\xi_{21}=0$ and $\xi_{22} \neq 0$ as only $t_{2}$ accounts for ECCD. All quantities on the right-hand side of (2),(3),(4) depend on $i$. Therefore, equation (1) can be re-written as

$$
0=\xi_{0 i}+c_{\mathrm{sat}} \xi_{1 i}+c_{\mathrm{stab}} \xi_{2 i}+\varepsilon
$$

where $\varepsilon$ is the deviance from $\frac{\tau_{\mathrm{s}}}{r_{\text {res }}} \frac{d W}{d t}=0$ and $\langle\varepsilon\rangle=0,\left\langle\varepsilon^{2}\right\rangle=\sigma^{2}$ where $\sigma^{2}$ is its variance. Ideally, $\frac{d W}{d t}=0$ at $t_{\mathrm{sat}}$ and $t_{\mathrm{ECCD}}$ in case of marginal stabilisation. In fact, it is assumed $\sigma^{2}=0.01$ for all discharges, which corresponds to an uncertainty in the evaluation of $\frac{d W}{d t}$ of about $10 \%$. By assuming a normally distributed error, the estimation of $c_{\text {sat }}$ and $c_{\text {stab }}$ corresponds to the minimisation of $\chi^{2}$ defined as:

$$
\frac{1}{2} \chi^{2}=\frac{1}{2} \sum_{i=1}^{2}\left(\frac{\xi_{0 i}+c_{\mathrm{sat}} \xi_{1 i}+c_{\mathrm{stab}} \xi_{2 i}}{\sigma_{i}}\right)^{2}
$$

In order to minimise $\chi^{2}$, the derivative with respect to $c_{\text {sat }}$ and $c_{\text {stab }}$ has to be 0 . By defining the following quantities:

$$
\begin{aligned}
& \beta_{11}=\sum_{i} \frac{\xi_{1 i}^{2}}{\sigma_{i}^{2}} ; \beta_{02}=\sum_{i} \frac{\xi_{0 i} \xi_{2 i}}{\sigma_{i}^{2}} ; \beta_{12}=\sum_{i} \frac{\xi_{1 i} \xi_{2 i}}{\sigma_{i}^{2}} \\
& \beta_{22}=\sum_{i} \frac{\xi_{2 i}^{2}}{\sigma_{i}^{2}} ; \beta_{01}=\sum_{i} \frac{\xi_{0 i} \xi_{1 i}}{\sigma_{i}^{2}}
\end{aligned}
$$

the system of equations to be solved simultaneously in order to determine $c_{\text {sat }}$ and $c_{\text {stab }}$ is

$$
\begin{aligned}
& c_{\text {sat }} \beta_{11}+c_{\text {stab }} \beta_{12}=\beta_{01} \\
& c_{\text {sat }} \beta_{12}+c_{\text {stab }} \beta_{22}=\beta_{02}
\end{aligned}
$$

which leads to the solutions

$$
\begin{aligned}
& c_{\text {sat }}=\frac{\beta_{01} \beta_{22}-\beta_{02} \beta_{12}}{\beta_{11} \beta_{22}-\beta_{12}^{2}} \\
& c_{\text {stab }}=\frac{\beta_{02} \beta_{11}-\beta_{01} \beta_{12}}{\beta_{11} \beta_{22}-\beta_{12}^{2}}
\end{aligned}
$$

At $t_{\text {sat }}$ where no ECCD was applied $\left(\xi_{21}=0\right), c_{\text {sat }}$ can be determined simply by $c_{\text {sat }}=\frac{\beta_{01}}{\beta_{11}}$. Equivalent formulas can be derived for the uncertainties of $c_{\mathrm{sat}}$ and $c_{\text {stab }}$ from equation (6). However, these uncertainties do only reflect the uncertainty in $\frac{d W}{d t}$ but do not encounter the 
correlation and the uncertainties on the other numerous physical quantities. To estimate full uncertainties of the coefficients applying Gaussian error propagation including all uncertainties is cumbersome and does not even include correlations. Therefore, a probabilistic approach is used.

\subsection{Bayesian approach to the Modified Rutherford equation}

To estimate $c_{\text {sat }}$ and $c_{\text {stab }}$, their uncertainties and correlations, from a comprehensive statistical description of all measured data and modelling parameters including parameter dependencies and constraints a probabilistic approach based on Bayesian Probability Theory is applied [7]. The Bayesian Theorem states that a posterior PDF of the event c given the event $\mathrm{B}, P(c \mid B)$, can be calculated by multiplying the prior PDF $P(c)$ by the likelihood $P(B \mid c)$ that event $\mathrm{B}$ will occur if $\mathrm{c}$ is true:

$$
P(c \mid B)=\frac{P(B \mid c) P(c)}{P(B)}
$$

The Bayesian theory is applied to the MRE written in the form of equation (1). $\bar{b}$ is the set of measured data with uncertainties and the parameters of interest $\bar{c}$ are $c_{\text {sat }}$ and $c_{\text {stab. The }}$ quantities $\bar{B}$ are the true quantities to be measured (e. g. $W_{\mathrm{sat}}, L_{\mathrm{q}}, j_{\mathrm{BS}}$, etc.) which are unknown. The marginal probability distribution for $c_{\text {sat }}$ and $c_{\text {stab }}$ can be obtained by applying the marginalisation theorem and the product rule of BPT,

$$
\begin{aligned}
p(\bar{c} \mid \bar{b}, \bar{\sigma}) & =\int d \bar{B} p(\bar{c}, \bar{B} \mid \bar{b}, \bar{\sigma}) \\
& =\int d \bar{B} \frac{p(\bar{b} \mid \bar{c}, \bar{B}, \bar{\sigma}) p(\bar{c}, \bar{B})}{p(\bar{b} \mid \bar{\sigma})} \\
& \propto \int d \bar{B} p(\bar{b} \mid \bar{B}, \bar{\sigma}) p(\bar{c} \mid \bar{B}) p(\bar{B})
\end{aligned}
$$

where the integral is calculated using Monte-Carlo simulation. $p(\bar{b} \mid \bar{B}, \bar{\sigma})$ is the product of the likelihood functions of all the measured quantities, the probability $p(\bar{c} \mid \bar{B})$ is given by the physical model (the MRE in this case) and $P(\bar{B})$ quantifies the dependencies and constraints characterising the physical values $\bar{B}$. The likelihood for each physical quantity is assumed to be a normal distribution:

$$
P\left(b_{i} \mid B_{i}, \sigma_{\mathrm{B}_{\mathrm{i}}}\right)=\frac{1}{\sqrt{2 \pi} \sigma_{B_{i}}} \exp ^{-\frac{1}{2}\left(\frac{b_{i}-B_{i}}{\sigma_{B_{i}}}\right)^{2}}
$$

The joint likelihood probability is given by

$$
p(\bar{b} \mid \bar{B}, \bar{\sigma})=\prod_{i} p\left(b_{i} \mid B_{i}, \sigma_{\mathrm{B}_{\mathrm{i}}}\right)
$$

The probabilistic method applied to the MRE has several advantages compared to a standard Gaussian error propagation method due to the complex structure of the MRE. It allows one to provide a comprehensive statistical description of all uncertainties, to consider the physics constraints and the correlations present in the model, and to make a sensitivity study on the full model to identify which are the most influencing quantities. The term $p(\bar{B})$ accounts for 
the physical constraints (positivity, thresholds, etc...) and for the automatic propagation of errors for each quantity $B_{N-1}$ which depends on the quantity $B_{N}$ :

$$
P(\bar{B})=P\left(B_{1} \mid B_{2}, \ldots\right) * P\left(B_{2} \mid B_{3} \ldots . .\right) * P\left(B_{N}\right)
$$

For example, starting from the likelihood of $j_{\mathrm{BS}}$ a sample is drawn subject to the imposed constraints (e.g. being a positive quantity). Successively, a second quantity like $j_{\mathrm{ECCD}}$ is sampled from its likelihood whose acceptable values need to fulfil the constraint $j_{\mathrm{ECCD}} / j_{\mathrm{BS}}>$ 0.3 .

After having sampled all the physical quantities $c_{\text {sat }}$ and $c_{\text {stab }}$ are determined from equation (10) and (11). The process of sampling a complete set of physical quantities and the subsequent calculation of $c_{\mathrm{sat}}$ and $c_{\mathrm{stab}}$ is repeated. From a large number of $c_{\mathrm{sat}}$ and $c_{\mathrm{stab}}$ values one calculates a 2-dimensional histogram which directly approximates the marginal distribution $p(\bar{c} \mid \bar{b}, \bar{\sigma})(13)$.

For the statistical analysis each quantity has been randomised with a sample number of 50000, according to the distribution of their uncertainties. This sample number is large enough to obtain sufficiently smooth marginal probability distributions. Whereas a sample number of 10000 has been tested to be the lower limit for the creation of smooth probability distributions. Marginal probability distributions for $c_{\text {sat }}$ and $c_{\text {stab }}$ are calculated for the single discharges individually as well as for all discharges jointly to obtain a unique value for $c_{\text {sat }}$ and $c_{\text {stab }}$ for the full database.

Therefore, the probabilistic approach for the MRE consists of the following steps:

(i) sample random values for $\bar{B}$ from probability distributions $p(\bar{b} \mid \bar{B}, \bar{\sigma}) \cdot p(\bar{B})$ for individual discharges starting from the collected data $\bar{b}$

(ii) calculate the marginal posterior probability distribution $p\left(c_{\mathrm{sat}}, c_{\mathrm{stab}} \mid \bar{b}, \bar{\sigma}\right)$ for each single discharge by generating histograms with the collected samples

(iii) calculate the marginal posterior probability distribution $p\left(c_{\mathrm{sat}}, c_{\mathrm{stab}} \mid \bar{b}, \bar{\sigma}\right)$ considering all the discharges simultaneously

(iv) calculate the mean values $\left\langle c_{\text {sat }}\right\rangle,\left\langle c_{\text {stab }}\right\rangle$ and the standard deviations $\Delta_{c_{\text {sat }}}, \Delta_{c_{\text {stab }}}$ from the marginal probability distributions for each single discharge as well as for all discharges together

(v) carry out a sensitivity study on the quantities $\bar{B}$ present in the MRE (see section 6.3)

Summarising, the probabilistic method is based on the $\chi^{2}$ approach for the MRE concerning the determination of the coefficients for given physical quantities. Additionally, the probabilistic method allows for arbitrary error distributions, model parameter correlations and boundary constraints. Therefore, a sensitivity study within the probabilistic approach with respect to major error sources improves the classical $\chi^{2}$ approach due to the complex dependencies.

\section{Results}

Considering the 23 discharges taken into account, the 2 time points necessary for the analysis and the 13 quantities which are considered within their uncertainties, the number of uncertain 

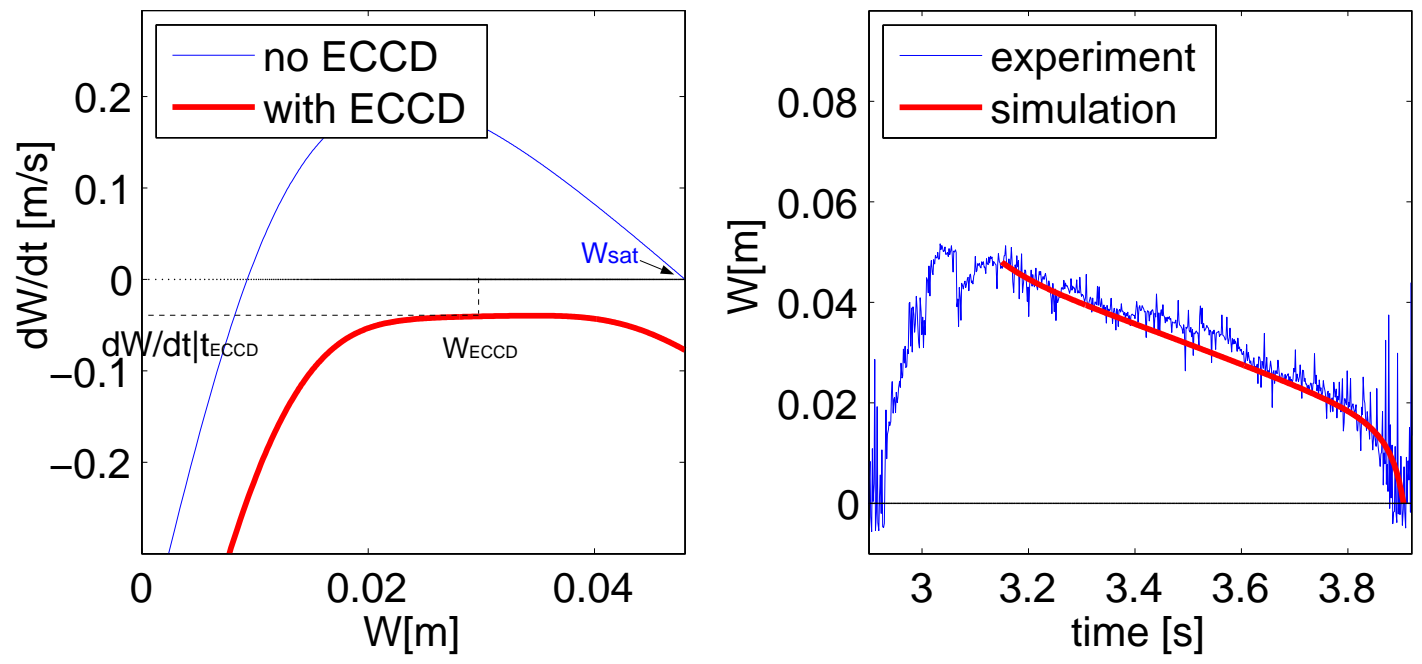

Figure 9. Phase diagram obtained from the MRE (left) and integrated MRE (right). Starting from the saturated island width $W_{\text {sat }}$ simulation of the island width decay is carried out by including the ECCD term in the MRE and by optimising the value of $c_{\text {stab }}$. In the experiment injection of ECCD current starts at $\sim 3.2 \mathrm{~s}$. Indicated are also the growth rate $d W /\left.d t\right|_{t_{\mathrm{ECCD}}}$ and the corresponding island width $W_{\mathrm{ECCD}}$.

model parameters in equation $(1)$ is $\approx N_{\text {discharges }} * 2 * 13 \approx 600$.

\subsection{Results for $c_{\mathrm{sat}}$ and $c_{\mathrm{stab}}$ with the $\chi^{2}$ method}

In [13] $c_{\text {sat }}$ and $c_{\text {stab }}$ have been evaluated for a set of experimental discharges collected at ASDEX Upgrade and JT-60U. The fitting method consists of a $\chi^{2}$ misfit minimisation (details are given in section 5.1) based on the measurement of the local quantities present in the MRE and the comparison between a simulated island width decay and the experimental one. $c_{\mathrm{sat}}$ has been evaluated at saturation time $t_{\text {sat }}$ when $d W / d t=0$ and $W=W_{\text {sat }} . c_{\text {stab }}$ has been evaluated during the stabilisation time when the island width decays until it becomes zero at $t_{\mathrm{ECCD}}$. The former makes use of the equation $c_{\mathrm{sat}}=\frac{\Delta^{\prime}}{\Delta_{\mathrm{BS}}^{\prime}+\Delta_{\mathrm{GGJ}}^{\prime}}$. The latter is determined by direct comparison with the experimental evolution. Both the phase diagram and the simulation optimised to the experimental curve of an NTM stabilisation discharge are shown in figure 9. The average values of the two coefficients $c_{\text {sat }}$ and $c_{\text {stab }}$ obtained with this method are:

$$
\begin{aligned}
& c_{\text {sat }}=0.81 \pm 0.13 \\
& c_{\text {stab }}=0.68 \pm 0.22
\end{aligned}
$$

All plasma quantities are kept constant without errors [13]. The uncertainties reflect the scatter of the coefficient values estimated from the different discharges.

\subsection{Results for $c_{\mathrm{sat}}$ and $c_{\mathrm{stab}}$ with the probabilistic method}

The results obtained for the marginalised posterior probability distributions for $c_{\text {sat }}$ and $c_{\text {stab }}$ over a single discharge and over all the discharges are obtained in terms of 2-dimensional 

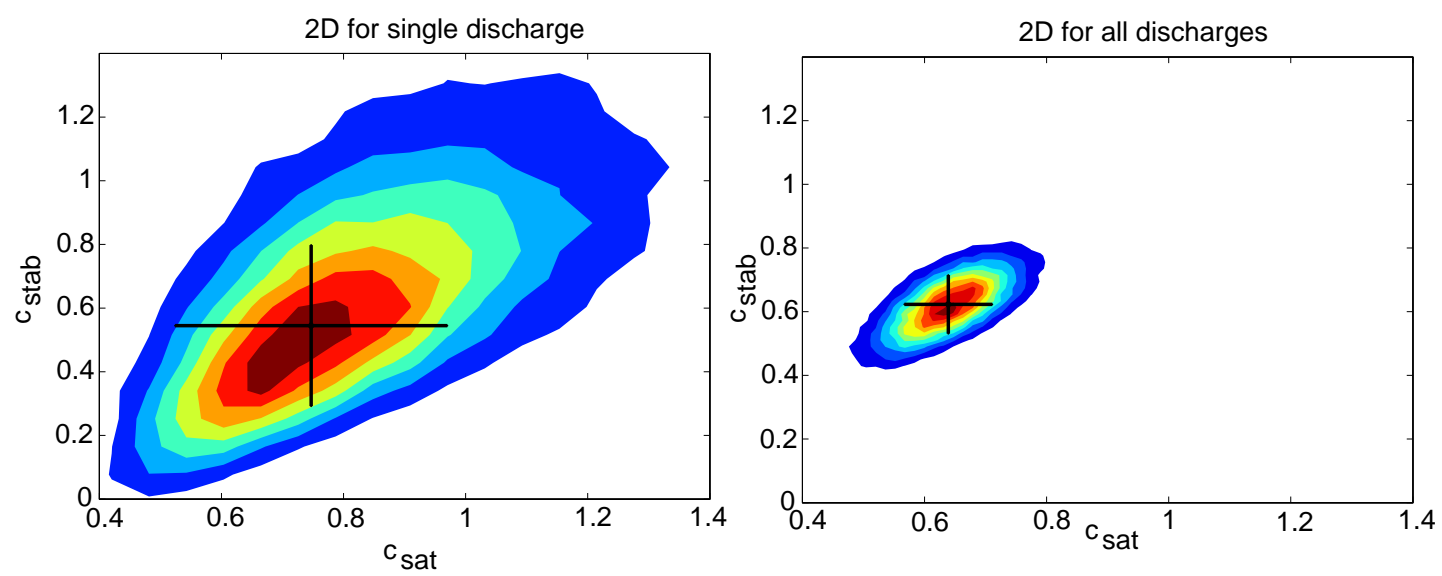

Figure 10. 2D marginal posterior probability distribution for one discharge (left) and for all discharges (right). Overplotted are the mean values for $\left(c_{\mathrm{sat}}, c_{\mathrm{stab}}\right)$ with their error bars

diagrams and are shown in figure 10. The cross describes the mean values and standard deviations of the marginal PDF. The results show that:

- The PDF of $c_{\text {sat }}$ and $c_{\text {stab }}$ obtained for each single discharge is centred around a value which agrees with the one calculated with the standard $\chi^{2}$ approach. Since the mean value of the PDF does not necessarily have to coincide with the maximum value of the $\chi^{2}$ method, this result shows that neglecting in the $\chi^{2}$ method the statistical uncertainties, the constraints and the correlations of the many physical quantities does not deteriorate the estimated values significantly.

- The PDF of $c_{\text {sat }}$ and $c_{\text {stab }}$ obtained by marginalising over all the discharges is centred around a mean value $\left(c_{\text {sat }}=0.64 \pm 0.07\right.$ and $\left.c_{\text {stab }}=0.62 \pm 0.09\right)$ which is slightly different but consistent with the value found from the $\chi^{2}$ calculation (e.g. $c_{\text {sat }}=$ $0.81 \pm 0.13$ and $c_{\text {stab }}=0.68 \pm 0.22$ ).

- The difference between the probabilistic and the classical results increases if the standard deviations assumed in the Monte-Carlo process is increased. If the uncertainties are reduced dramatically, the overall PDF for all the discharges agrees with the value obtained in ref. [13]. This suggests that the non-linearity of the MRE is responsible for the difference. This also suggests that for some quantities a skewed error probability distribution might better represent the distribution of its values compared to the normal distribution which has been assumed here.

- The uncertainties for the coefficients obtained with the probabilistic method are smaller than the uncertainties from the $\chi^{2}$ method, although the $\chi^{2}$ method is not explicitely incorporating uncertainties on the physical quantities. The reason is that with the $\chi^{2}$ method the uncertainties are determined from the scatter of the estimated coefficients for single discharges. This scatter implicitly includes the measurement errors on the physical parameters. The larger uncertainties arise from the missing statistical quantification of the physical parameters and the missing constraints and correlations. Providing more information results in more reliable estimates. 
- As expected, the standard deviation of the PDF for $c_{\mathrm{sat}}$ and $c_{\mathrm{stab}}$ for marginalising over all the discharges is smaller $(\sigma=10 \% \div 15 \%)$ compared to the values obtained for single discharges $(\sigma=30 \% \div 40 \%)$, although one would expect a somewhat larger uncertainty reduction than a factor of 3 from 23 discharges. The reason might be a natural scatter of the discharge properties which is not included in the measurement uncertainties of the physical quantities.

- The PDF over a single discharge as well as for the combined discharges show a clear correlation between $c_{\text {sat }}$ and $c_{\text {stab }}$. They are positively correlated. Overestimation of one coefficient results in simultaneous overestimation of the other one.

The results show that the classical approach provides reasonable estimation values and uncertainties for the MRE coefficients. The conceptual drawbacks of the classical approach were overcome with the probabilistic method. The probabilistic approach results in more reliable estimates due to the additional information which can be easily incorporated with the Bayesian method. An important advantage of BPT is that all relevant information can and has to be quantified explicitely which provides an ideal framework for scientific reasoning.

The amount of ECRH power necessary to stabilise NTMs is directly proportional to the ratio $c_{\text {sat }} / c_{\text {stab }}$ (e.g. $P_{\mathrm{ECRH}} \propto j_{\mathrm{ECCD}} / j_{\mathrm{BS}} \propto c_{\text {sat }} / c_{\text {stab }}$ [14]). Therefore, the uncertainty on $c_{\text {sat }}$ and $c_{\text {stab }}$ directly influences the ECRH power necessary for ITER. In [13], the minimum amount of ECRH power is estimated to be about $10 \mathrm{MW}$. From the error analysis of this work one can estimate an uncertainty of $\pm 2 \mathrm{MW}$ on these calculations. This is indeed a comforting result since in ITER, an ECRH system delivering a maximum of $20 \mathrm{MW}$ of ECRH power is foreseen.

\subsection{Sensitivity analysis}

As mentioned in the previous section, not all the quantities present in the MRE have been randomised according to their uncertainty since their negligible uncertainty does not affect the evaluation of $c_{\text {sat }}$ and $c_{\text {stab }}$ significantly. Nevertheless, for the other quantities considered in the statistical analysis it is interesting to study which quantity has the most effect on the overall uncertainty. In fact, it is relevant to understand how precise the measurements on such quantities need to be in order to have robust results for the MRE coefficients. In order to carry out such a sensitivity study only one relevant quantity at a time is considered by switching off the other quantities' uncertainties. Statistically, this means to marginalise the probability distribution over one of the quantities given that the other quantities are kept constant, as for example $L_{\mathrm{q}}$ :

$$
p\left(c_{\mathrm{sat}}, c_{\mathrm{stab}} \mid \bar{d}\right)=\int d L_{\mathrm{q}} p\left(c_{\mathrm{sat}}, c_{\mathrm{stab}}, L_{\mathrm{q}} \mid \bar{d}\right)
$$

The results of the sensitivity analysis are shown in figure 11 where each quantity is randomised over the uncertainties listed in table 2. $W_{\mathrm{sat}}, j_{\mathrm{BS}}$ and $L_{\mathrm{q}}$ are found to be the most crucial ones for the determination of $c_{\text {sat }}$. This is expected as physically the saturated island size $W_{\text {sat }}$ and the bootstrap current density $j_{\mathrm{BS}}$ are proportional. Both depend on the pressure of the plasma. The strong dependency on the safety factor gradient length, on the other hand, is related to the 

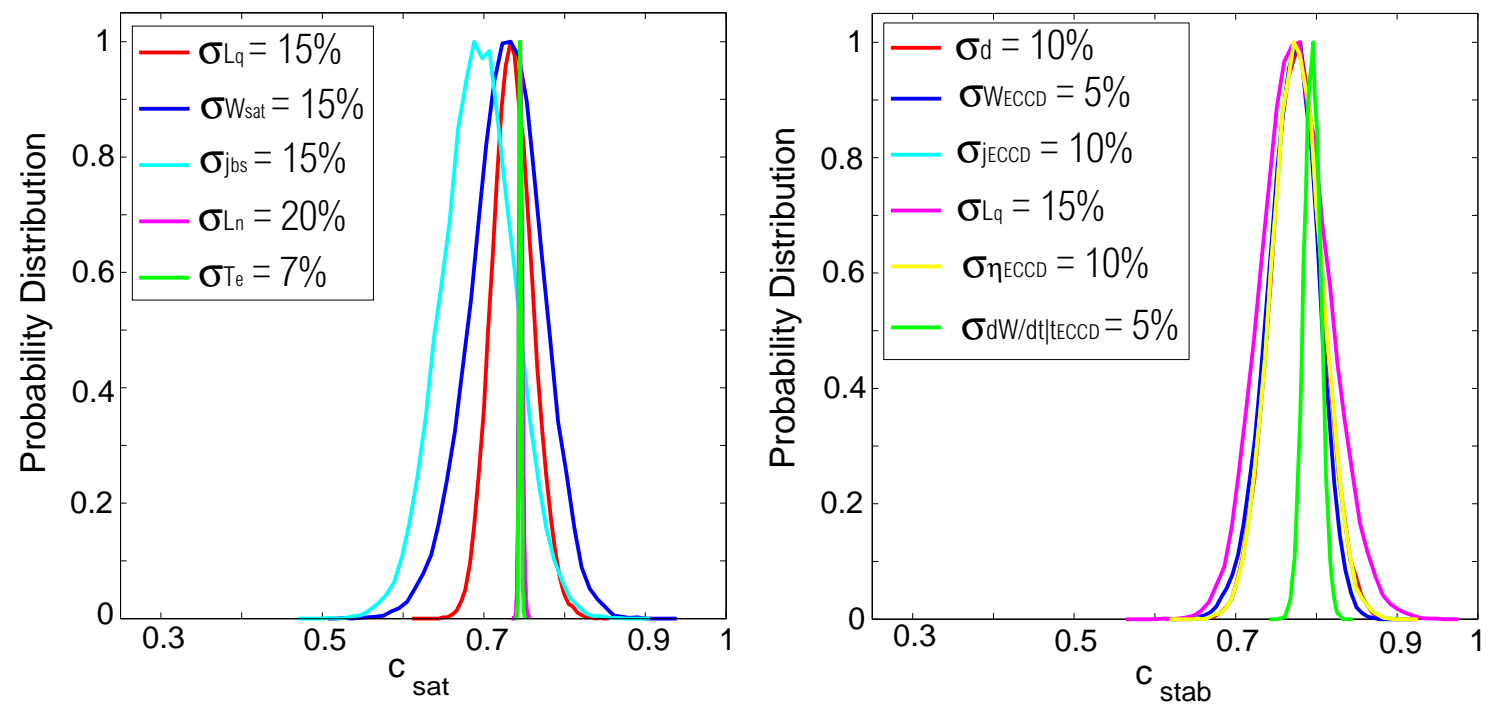

Figure 11. Marginal probability distributions for $c_{\text {sat }}$ and $c_{\text {stab }}$ for various uncertain physical quantities where all the other uncertainties are assumed to vanish.

effect of the shear on the island width. The uncertainty of $L_{\mathrm{ne}}$, which is quite large, has almost no effect since it enters explicitly in the MRE only in the curvature term via the pressure gradient length $L_{\mathrm{p}}=p / p^{\prime}$. For $c_{\text {stab }}$ nearly all of the quantities affect the evaluation in a similar way. Only the uncertainty of the quantity $d W / d t \mid t_{\mathrm{ECCD}}$ has little impact on the width of the probability distribution. Additionally, it is interesting to notice that some quantities like $j_{\mathrm{BS}}$ and $W_{\mathrm{ECCD}}$ tend to shift the centre of the probability distribution towards the left as their uncertainty is increased. This is due to the non-linear dependence of $c_{\text {stab }}$ on these quantities.

Summarising, the sensitivity analysis shows clearly which physical parameters are most important to obtain efficient improvements in estimating the MRE coefficients. Additionally, an improved statistical description for those parameters employing non-Gaussian probability distributions might be most beneficial.

\section{Summary and conclusion}

The Modified Rutherford Equation is routinely used to study the evolution of magnetic islands associated to the presence of NTMs in fusion devices. To improve the description of NTMs with the MRE, its expression needs to be verified and its free coefficients $c_{\text {sat }}$ and $c_{\text {stab }}$ need to be determined from the experiment. It is important to obtain estimates of these coefficients with high precision as the estimate of the amount of ECCD power required for NTM stabilisation in ITER directly depends on their values as well as on their uncertainties. Therefore, to calculate the error bars of $c_{\text {sat }}$ and $c_{\text {stab }}$ a new technique is developed which makes use of the Bayesian Probability Theory. Monte-Carlo simulations are applied to generate probability distributions for $c_{\text {sat }}$ and $c_{\text {stab }}$ starting from the probability distributions of all the physical quantities present in the MRE which quantify the measured or derived uncertainties, the correlations and the constraints. 
The resulting standard deviations of $c_{\mathrm{sat}}$ and $c_{\mathrm{stab}}$ for single discharges are between $30 \%$ and $40 \%$. An analysis of the combined set of discharges results in mean values of $c_{\text {sat }}=0.64 \pm 0.07$ and $c_{\text {stab }}=0.62 \pm 0.09$. In this case the uncertainty decreases to about $12 \%$. As a consequence the minimum ECRH power requirements for NTM stabilisation in ITER can be estimated to be $10 \pm 2 \mathrm{MW}$. A sensitivity study shows that the most important three quantities needed to be estimated with high precision for a good evaluation of $c_{\text {sat }}$ and $c_{\text {stab }}$ are the saturated island size $W_{\text {sat }}$, the bootstrap current density $j_{\mathrm{BS}}$ and the local shear value $L_{\mathrm{q}}$.

The probabilistic approach is not restricted to the MRE analysis but can be applied to any problem with multiple uncertainties in physical quantities.

\section{References}

[1] A. I. Smolyakov, Plasma Physics and Controlled Fusion, Vol. 46, 3, L1-L6 (2004)

[2] A. Peeters, Plasma Physics and Controlled Fusion, Vol. 42, B231-B242, (2000)

[3] H. Zohm, Nuclear Fusion, Vol. 41, 2, 197-202, (2001)

[4] H. Lütjens et al., Plasma Physics and Controlled Fusion, Vol. 43, A339-A348, (2001)

[5] M. Maraschek e al., Fusion Energy, (Proc. 20th Int. Conf. Vilamoura, 2004) (Vienna: IAEA) CD-ROM $E X / \&-2$ and http://www-naweb.iaea.org/napc/physics/fec/fec2004/datasets/index.html

[6] A. Isayama, Nuclear Fusion, Vol. 43, 10, 1272-1278, (2003)

[7] M. Goldstein, 'Bayes Linear Statistics, Theory and Methods', Wiley Publisher, (2007)

[8] R. Fischer and A. Dinklage, In Da Ruan, J. Montero, J. Lu, L. Martinez, P. D'hondt, and E. E Kerre, editors, Computational Intelligence in Decision and Control, Proceedings of the 8th International FLINS Conference, Vol. 1, 37-42, Singapore, 2008. World Scientific.

[9] R. Fischer and A. Dinklage, Rev. Sci. Instrum., 75: 4237-4239, (2004)

[10] L. Urso et al., J. Phys. Conf. Ser. 25, 266-273, (2005)

[11] Q. Yu, Physics of Plasmas, Vol. 11, 1, 140-150 (2004)

[12] J. Wesson, 'Tokamaks', $2^{\text {nd }}$ edition, Oxford University Press, (1997)

[13] L. Urso et al., Nuclear Fusion, Vol. 50, 025010

[14] H. Zohm et al., Plasma Physics and Controlled Fusion, Vol. 49, B341-B347, (2007)

[15] C. C. Hegna and J. D. Callen, Physics of Plasmas, Vol. 4, 2940, (1997) 